\title{
Marcos interpretativos: reelección presidencial y la figura de Álvaro Uribe Vélez en la revista Semana ${ }^{1}$
}

\section{Andrés Felipe Yepes-Charry²}

Recibido: 2014-04-24

Enviado a pares: 2014-04-28

DOI: 10.5294/pacla.2015.18.2.9
Aprobado por pares: 2014-06-01

Aceptado: 2014-08-19

Para citar este artículo / To reference this article / Para citar este artigo

Yepes-Charry, A.F. Junio de 2015. Marcos interpretativos: reelección presidencial y la figura de Álvaro Uribe Vélez en la revista Semana. Palabra Clave 18(2), 537-562. DOI: 10.5294/pacla. 2015.18.2.9

\section{Resumen}

Este artículo busca mostrar cómo presentó la revista Semana el proceso de reforma constitucional que permite la reelección presidencial inmediata en Colombia, y cuál fue el significado que le otorgó. Así pues, en el primer segmento se presenta y recupera una serie de elementos relacionados con el tema de la reelección presidencial. En el segundo segmento se exponen los marcos interpretativos textuales (framing) y los paquetes de significado de cara a abordar la noticia (texto/información) y se revisan los diferentes artículos que fueron publicados en la revista Semana relacionados con el tema de la reelección presidencial y la figura de Álvaro Uribe Vélez. En el último

1 Una versión previa de este artículo fue presentada en la Jornada de Jóvenes Investigadores del V Congreso Latinoamericano de Opinión Pública WAPOR 2012, Septiembre 19-21, Bogotá.

Este artículo no hubiera sido posible sin el apoyo y colaboración de Paula Cobo y de Alejando Ángulo Novoa, S. J. A ellos mi más sincera gratitud.

2 Magíster en comunicación y politólogo de la Pontificia Universidad Javeriana. Colombia. andresfelipeyepes@gmail.com 
segmento se presentan los hallazgos encontrados en relación con la información presentada por parte de la revista Semana y el tema de la reelección.

\section{Palabras clave}

Información política, medios de información, prensa (Fuente: Tesauro de la Unesco).

\section{Marcos Interpretive: Presidential Reelection and the Figure of Alvaro Uribe Velez in Semana Magazine}

\section{Abstract}

This article seeks to show how Semana magazine presented the process of constitutional reform that allows immediate presidential reelection in Colombia, and what was the meaning that was given. Thus, in the first segment it is presented and recovered a number of items related to the topic of presidential reelection. In the second segment textual interpretative frames (framing) and packages meant facing addressing the news (text / information) and different articles published in the Semana magazine related to the theme of the presidential reelection are reviewed and the figure of Alvaro Uribe Velez. In the last segment of the findings in relation to the information presented by Semana magazine and the issue of reelection are presented.

\section{Keywords}

Political information, media, press (Source: Unesco Thesaurus). 


\section{Marcos interpretativos: reeleição presidencial e a figura de Álvaro Uribe Vélez na revista Semana}

\section{Resumo}

Este artigo pretende mostrar como a revista Semana apresentou o processo de reforma constitucional que permite a reeleição presidencial imediata na Colômbia e qual foi o significado que outorgou a ele. Para isso, na primeira seção, apresenta e recupera-se uma série de elementos relacionados com o tema da reeleição presidencial. Na segunda seção, expõem-se os marcos interpretativos textuais (framing) e os pacotes de significado a fim de abordar a notícia (texto/informação); além disso, revisam-se os diferentes artigos que foram publicados na revista Semana relacionados com o tema da reeleição presidencial e a figura de Álvaro Uribe Vélez. Na última seção, apresentam-se as constatações referentes à informação apresentada por parte da revista Semana e o tema da reeleição.

\section{Palavras-chave}

Informação política, meios de informação, imprensa. (Fonte: Tesauro da Unesco). 


\section{La reelección presidencial inmediata en Colombia}

La historia constitucional de Colombia ha mantenido el régimen presidencialista con una serie de modificaciones en lo referente a las facultades de la cabeza del poder ejecutivo. Desde la constitución de 1821 (Restrepo Piedrahita, 1988) hasta la de 1991, la historia política se caracteriza por la concentración del poder en manos del ejecutivo. Predomina la posición del presidente como jefe de Estado, de Gobierno, suprema autoridad administrativa, comandante y jefe de las Fuerzas Militares. El presidente de la República es elegido por un periodo de cuatros años; desde el año 2006 puede ser reelegido inmediatamente por un periodo adicional (tras la aprobación del Acto Legislativo 02 de 2004, por el cual se reforma la Constitución para permitir la reelección presidencial inmediata).

La reelección presidencial inmediata ha sido uno de los temas de reforma a la Constitución que han agotado el "papel” en los medios de comunicación del país en los últimos años. La información sobre este tema ha tenido varios momentos: el primero recoge los elementos relacionados con la reforma como tal; el segundo, con la campaña electoral del presidentecandidato Álvaro Uribe Vélez en 2006; el tercero tiene que ver con la entrega de notarías a algunos congresistas tras el apoyo a la iniciativa a la reforma; el cuarto, con la campaña electoral de 2014, en cuya primera vuelta participaron Martha Lucía Ramírez (Partido Conservador Colombiano), Clara López Obregón (Polo Democrático Alternativo), Enrique Peñalosa (Partido Verde), Óscar Iván Zuluaga (Centro Democrático) y el presidente-candidato Juan Manuel Santos Calderón (Unidad Nacional: Cambio Radical, Partido Social de Unidad Nacional -Partido de la U- y Partido Liberal), y en segunda vuelta se definió el presidente entre el candidato del Centro Democrático y el de la Unidad Nacional; el último, con la intención de reformar la Constitución para acabar la reelección presidencial inmediata, según dio a conocer Juan Manuel Santos tras ganar en segunda vuelta y reiteró luego durante la instalación de la legislatura 2014-2015.

La reelección se tornó decisiva en el primer mandato del presidente Uribe Vélez (2002-2006), aunque en principio no fue bandera de campaña ni de gobierno. El primer acto del gobierno Uribe Vélez fue la propues- 
ta de un referendo anticorrupción; con el pasar del tiempo, mantenerse en el poder por otro periodo desbancó en la agenda legislativa algunas de las principales propuestas, como la reforma pensional y laboral, y terminó por convertirse no solo en asunto prioritario sino que copó todos los espacios posibles en las preocupaciones de la política del país y marcó el trecho restante de su mandato.

El acontecimiento de la reelección dividió al país en torno al tema e hizo que se adoptaran dos posturas acerca de la reelección presidencial, una a favor y una en contra. Para la primera postura, los argumentos eran: 1) la reelección permite a los ciudadanos juzgar los mandatos presidenciales y 2) Uribe Vélez debe mantenerse en el poder por otros cuatro años por sus características políticas o personales y debe darse continuidad a la política de Seguridad Democrática.

Para la segunda postura, los argumentos se centraban principalmente en que, aunque se a estuviera de acuerdo con el mecanismo, el proyecto de reelección se había hecho a la medida para favorecer al presidente.

Durante el episodio de la reforma constitucional no era difícil conocer qué estaba ocurriendo en los debates del Congreso, cuáles eran los conceptos sobre la reelección que se estaban usando en la discusión y cuál era el papel y el fallo de la Corte Constitucional, que era la que en últimas decidía qué iba a pasar, porque los medios de comunicación realizaron un cubrimiento muy amplio, pues la reelección modificaba el panorama político nacional.

Los últimos años en el mundo de las revistas han estado marcados por el auge de productos relacionados con temas de farándula, entretenimiento y moda, pero esta tendencia no se refleja en revistas de actualidad, opinión y política. Es por esto que la revista Semana, teniendo en cuenta la información de lecturabilidad del Estudio General de Medios (EGM) -realizado semestralmente por la Asociación Colombiana de Investigaciones en Medios (ACIM)-, se ubicaba durante el primer gobierno de Uribe Vélez como la primera y hoy como la única revista de este tipo con un número nada despreciable de lectores, que fluctúa entre 800 y 900 000. Esta 
cifra se ha mantenido en el tiempo, pese a que la revista Semana se ha movido entre los primeros lugares en las distintas olas del EGM, compartiendo los primeros lugares con revistas de farándula y entretenimiento ( $T v$ y Novelas, SoHo, 15 Minutos y, recientemente, VEA). Para el momento de clímax de la reforma que permitiría la reelección presidencial inmediata en Colombia, la información de la segunda ola de 2006 muestra que la revista Semana ocupaba el tercer lugar de lecturabilidad, con 850300 lectores, y la edición digital era el segundo medio electrónico de Colombia más consultado en el mundo, después del periódico El Tiempo. La revista Semana solo se encontraba por debajo de las revistas Tv y Novelas -de circulación quincenal-y SoHo -mensual-, que tenían 1028200 y 994000 lectores respectivamente.

Si bien los medios en Colombia tienen una historia que tiende a ser "gobiernista", es interesante mirar el caso de la revista Semana y la reforma que permitió la reelección presidencial inmediata en el país, no solo por lo mencionado anteriormente, sino también porque es sugestivo acercarse al tratamiento del tema por parte de la revista, que fue blanco de críticas en diferentes momentos por parte de Uribe Vélez, quien la acusa de dar información tendenciosa vinculada a su gobierno. En 2006, las afirmaciones que Uribe Vélez dio en este sentido fueron motivadas por la información publicada sobre el escándalo de las interceptaciones y seguimientos ilegales por parte del Departamento Administrativo de Seguridad (DAS), y en 2014 sus afirmaciones fueron provocadas por la publicación relacionada con las interceptaciones al equipo de negociación del gobierno en el proceso de paz que se adelanta en La Habana con la guerrilla de las FARC.

Para realizar el análisis se seleccionaron 18 ediciones de la revista Semana, en las que se encontraron 24 artículos directamente relacionados con la reelección presidencial y la figura del presidente Álvaro Uribe Vélez. Los artículos seleccionados hacen parte de la sección Nación/Política, y en todos los casos el medio omitió el nombre del redactor. El primero fue publicado el 28 de julio de 2003 (edición 1108) y el último, el 24 de octubre de 2005 (edición 1225). Se inició el análisis con el artículo del 28 de julio de 2003, pues en este se menciona por primera vez el tema de la reelección presidencial. 
Sin embargo, el primer artículo en el que se hace manifiesta la intención del Gobierno de apoyar la reelección fue publicado el 9 de febrero de 2004 (edición 1136). En él se menciona que la embajadora en España, Noemí Sanín, y el asesor presidencial Fabio Echeverri plantean la importancia de que Uribe permanezca por cuatro años más en el cargo. Los últimos artículos que se seleccionaron hacen parte del informe especial del 24 de octubre de 2005 (edición 1225), publicado tras el fallo de la Corte Constitucional sobre la reforma que permitiría la reelección presidencial inmediata. En general, los artículos escogidos son útiles en la medida en que presentan la totalidad del proceso desde el primer planteamiento de la reelección de Uribe Vélez hasta el fallo de la Corte Constitucional.

\section{Marcos interpretativos textuales y paquetes de significado}

En la investigación sobre la comunicación política se han desarrollado una serie de herramientas para abordar la noticia (texto/información), entre las cuales están los marcos interpretativos textuales o framing.

Han sido varias las definiciones desarrolladas sobre los marcos. Trabajos como los de Pan \& Kosicki (1993) y Reese (2001) son ilustrativos. Específicamente para el caso de este trabajo se tiene como referencia la siguiente definición: un marco es "una idea central que organiza y da significado a eventos relacionados con un tema" (Gamson \& Modigliani, citado en Pan \& Kosicki, 1993: 56), de tal forma que "un marco selecciona algunos aspectos de una realidad percibida que sobresalen, para así promover un problema particular, una definición, una interpretación causal, y una evaluación moral” (Entman, citado en Reese, 2001: 10).

Como elemento complementario al marco interpretativo, encuentro útil usar también los paquetes de significado (o packages, en inglés). Los paquetes de significado son "unidades interpretativas con una estructura interna que refuerzan un marco determinado y orientan la comprensión de un tema particular" (Gamson \& Modigliani, 1989: 3); es decir, los paquetes son un conjunto de interpretaciones que le dan significado a un tema. Estos paquetes ofrecen un número condensado de símbolos que sugieren 
las posiciones principales de las personas frente a un tema particular. Los paquetes son dinámicos y se adaptan para darles significado a nuevos hechos, ya que "todo tema político responde a una cultura y a un discurso cambiante con el transcurso del tiempo" (Gamson \& Modigliani, 1989: 1-2).

Así pues, los marcos caracterizan un tema y le dan significado, según el desarrollo que Pan \& Kosicki (1993: 58-62) le dan a las características y categorías del análisis de los marcos interpretativos textuales.

Las principales características de los marcos interpretativos textuales son: a) la consistencia de dispositivos simbólicos organizados que interactúan con la memoria de las personas para construir significado; b) la aceptación de la influencia natural de la formación del texto y el carácter polisémico de las noticias, lo cual puede llegar a ocasionar un cortocircuito cognitivo en la producción y consumo de las noticias; c) la validez del análisis recae en el proceso sistemático de recolectar información de la noticias e identificar los elementos significativos que pueden llegar a ser usados por los miembros audiencia, y d) no asumen la presencia de los marcos en las noticias independientemente de los lectores de los textos.

Las categorías de Pan \& Kosicki señalan cuatro dimensiones estructurales del discurso noticioso: la estructura sintáctica, la estructura del guión o escrito, la estructura temática y la estructura retórica.

La estructura sintáctica se caracteriza por describir los límites y reglas que permiten que las palabras se conviertan en oraciones. ${ }^{3}$ Por su parte, la estructura del guión o escrito está relacionada con el establecimiento de una secuencia de actividades y componentes de un evento que ha sido interiorizado como la representación mental estructurada de dicho evento. La estructura temática, por otro lado, hace referencia a que no todas las noticias son acciones o eventos orientados, puesto que algunas noticias

3 Al hacer referencia a la estructura sintáctica, Pan \& Kosicki retoman los planteamientos de Van Dijk, el cual enfatiza en el papel de la pirámide invertida, que consiste en la organización secuencial de los elementos estructurales (título, lead, eventos, contenido principal, cierre) y el poder significativo de estos elementos de manera descendente, es decir que el título es el elemento más importante para activar semánticamente conceptos en la mente de los lectores, mientras que el cierre es el de menor importancia (1993: 59). 
se centran en presentar un aspecto de un asunto y a la vez presentan una serie de eventos, acciones relacionadas con el tema, con objeto de soportar la hipótesis que el periodista presenta. Por último, la estructura retórica describe las elecciones de estilo hechas por los periodistas en relación con sus efectos planeados. En esta estructura es donde los planteamientos de Gamson \& Modigliani acerca de los componentes del marco se tornan relevantes (metáforas, ejemplos, frases del común, pinturas o esculturas e imágenes visuales); es decir que estos componentes ayudan a darles un significado a determinados conceptos y en algunos casos a hacerlos propios. Básicamente los marcos interpretativos textuales ayudan, persuaden o influyen en la forma en que las personas se acercan a un tema, lo estudian y lo recuerdan.

\section{Los marcos interpretativos y los paquetes de contenido en el caso de la reelección presidencial inmediata en Colombia}

A partir del análisis de los 24 artículos mencionados y a la luz de los planteamientos de Gamson identifico el siguiente marco:

\section{Marco predominante}

\begin{tabular}{|l|l|}
\hline Problema & Los mandatos presidenciales en Colombia deben ser juzgados por los ciudadanos. \\
\hline Causa & La Constitución Política de 1991 no permite la reelección presidencial. \\
\hline Solución & $\begin{array}{l}\text { El acto legislativo por el cual se permite la reelección presidencial y por el que se reforma la Constitución } \\
\text { de } 1991 \text { debe ser aprobado para permitir que los ciudadanos juzguen los mandatos presidenciales, de } \\
\text { modo que Álvaro Uribe Vélez pueda presentarse para ser reelegido y los ciudadanos puedan juzgar su } \\
\text { mandato mediante el voto. }\end{array}$ \\
\hline
\end{tabular}

\section{Paquetes de contenido}

\section{a. El presidente Álvaro Uribe Vélez es un hombre trabajador y un político diferente}

A partir de las siguientes citas se desarrolló el paquete de contenido mencionado: 
Álvaro Uribe es el único presidente de América Latina que no lo quieren tumbar sino reelegir. Colombia es, hasta ahora, en esta materia la excepción del continente. El rechazo de los países latinoamericanos a los sistemas partidistas tradicionales desembocó con el nuevo siglo con alternativas políticas improvisadas que tienen a casi todos nuestros vecinos al borde del abismo. (...) El único país que tuvo la suerte de que esa frustración se canalizara hacia algo serio fue Colombia. Hacia un hombre puritano, trabajador y conocedor del sistema políti$\underline{\mathrm{CO}^{4}}$. Un sistema que ha explorado en detalle como concejal, senador, alcalde, gobernador. $Y$ a pesar de ser un hijo de la clase política, Uribe se presentó como un disidente y coronó. Y eso es un milagro. En Colombia fue elegido, como símbolo de renovación política, un antipolítico que conoce como nadie la política (Semana, 28 de julio-4 de agosto, 2003: 24).

Uribe, el hombre personifica los valores de la gran mayoría de los colombianos que no son noticia: el trabajo y el sacrificio. Con ese aire de campesino, de ruana y sombrero, y recorriendo los lugares más recónditos del país, el Presidente está ahí a la mano. (...) en una cultura del dinero fácil y de los atajos el Presidente reivindica la ética del trabajo (...). Y cuando el poder presidencial no salía de los tapetes rojos de palacio o de la ciudad amurallada de Cartagena, Uribe acerca el poder a la gente y traslada la sede de gobierno a tierra caliento 0 ¡qué tal! A las narices de las FARC, en Arauca (p. 24).

La gente se siente más segura hoy y atribuye esa mejoría al empeño responsable, diario, del Presidente en velar porque la fuerza pública haga mejor su trabajo (Semana, 9-16 de febrero, 2004: 27).

En segundo lugar los partidarios de Uribe dicen que quisieran votar por él por su liderazgo personal y compromiso ( 26 por ciento) (Semana, 7-14 de junio, 2004: 40).

Esta última cita es relevante en la medida en que es el resultado de una encuesta por parte de la revista Semana y Gallup en las principales ciudades del país. El resultado de la encuesta, resumido en la frase citada, permite observar los factores que tienen en cuenta los ciudadanos para votar por Uribe.

Uribe es el líder ideal para ser reelegido porque "se ha preparado toda la vida para gobernar" (Semana, 19-26 de julio, 2004: 46).

4 El subrayado es intencional y pretende resaltar las figuras que soportan el paquete de contenido. 
El verdadero liderazgo de Uribe sobre la política de Seguridad Democrática es que la armó para que no dependiera de él. Se trata de una estrategia integral que interpreta las dificultades del conflicto interno y la escasez de recursos del Estado, pero que ha logrado fortalecer a las Fuerzas Armadas y profesionalizar a la tropa (Semana, 12-19 de septiembre, 2004: 44).

Álvaro Uribe es un mandatario responsable y comprometido y es de suponer que no tiene interés en abusar de la transformación institucional que ha promovido (Semana, 24-31 de octubre, 2005: 29).

Este paquete de contenido ofrece una serie de elementos que refuerzan la figura de Uribe Vélez, no solo en lo personal sino también como sujeto político, dándole atributos como el sacrificio, el compromiso y el trabajo. El sacrificio se muestra en los "actos" de abnegación inspirados por su vehemencia y su amor a la patria y en su empeño de responder a la obligación adquirida al ser elegido en 2002 como presidente para acabar con los múltiples males que agobian al país, principalmente la guerrilla. Pero la relación del presidente con el trabajo es la que articula dichos atributos, en la medida en que en el trabajo se encuentra la única salida a todos los problemas. El trabajo se ve en los resultados; la consigna de su gobierno era "trabajar, trabajar y trabajar”.

En lo político, se destaca el liderazgo y talante antipolítico del presidente. Esto se enfatiza al hacer explícito que combate la política tradicional, la cual ha sido causante de la corrupción y la crisis nacional. La caracterización de Uribe Vélez como disidente, al ser articulada con sus rasgos personales, hace que se presente como el hombre que necesita el país, puesto que representa al hombre trabajador e incansable que se entrega día y noche por la causa. Estas características lo presentan como un presidente diferente a los demás y con una política nueva.

Igualmente, Uribe Vélez es presentado como un presidente terrenal que, a diferencia de sus antecesores, es cercano a la gente, que no gobierna desde el Palacio de Nariño. Es un presidente cercano que entra en contacto con los colombianos. 
El encuentro del presidente con los ciudadanos no solo lo hace ver más cercano a la gente: también lo hacer ver como uno de ellos y más humano. Pese a ser una figura lejana, vestir ruana y sombrero lo lleva a camuflarse como un civil más, que sin embargo es el incansable presidente que se preocupa hasta por el más pequeño problema y no duerme hasta encontrarle solución, demostrando su latente preocupación de trabajo por la comunidad.

\title{
b. La lucha contra la corrupción y la politiquería es una característica del gobierno de Uribe Vélez
}

\begin{abstract}
Gran parte de la popularidad del Presidente Uribe radica precisamente en la percepción generalizada de que este Presidente, a diferencia de sus antecesores, estaba logrando gobernar sin cederles terreno a los políticos" (Semana, 9-16 de febrero, 2004: 26).
\end{abstract}

Al fin y al cabo casi uno de cada cuatro ciudadanos que lo apoyan lo hace porque confía en que no cede ante la politiquería (Semana, 7-14 de junio, 2004: 40).

Esta es la tercera razón de las que más motivan a la hora de votar por Uribe, y la cita es importante en la medida en que resume el resultado de una encuesta de Semana y Gallup y, de cierta forma, permite ver los diferentes motivos por los cuales llegarían a votar los ciudadanos por Uribe Vélez si se llegara a aprobar la reelección presidencial.

Este paquete de significado escenifica y refuerza una de las razones por las que en 2002 fue elegido Uribe Vélez: porque abogaba por la moralización de las costumbres políticas en un país en el que la política se usa para fines personales, en el que la política y los políticos han perdido legitimidad, en el que las promesas electorales no se cumplen y en el que el interés por la política es cada vez menor. En este contexto es vital contar con la figura de un presidente que rescata las virtudes de la política y los políticos. Esto es lo que la gente quiere y necesita oír. El paquete presenta a Uribe como un político diferente que rompe con la forma tradicional de hacer política. 
Adicionalmente, es importante resaltar que este paquete y el anterior presentan al presidente Uribe como un hombre de palabra que cumple lo que promete.

\section{c. La política de Seguridad Democrática ha sido exitosa, ha dado resultados, con la reelección se recuperará la continuidad, un elemento fundamental en políticas públicas}

Por eso quizás el mayor logro de Uribe en su primer año de gobierno es haber definido una estrategia de seguridad cuyo objetivo central no es perseguir al enemigo sino proteger al ciudadano (Semana, 28 de julio-4 de agosto, 2003: 25).

Si hay una razón principal por la cual quieren los colombianos que Uribe se quede es por sus éxitos en materia de seguridad. La gente se siente más segura hoy y atribuye esa mejoría al empeño responsable, diario, del Presidente en velar porque la fuerza pública haga mejor su trabajo (Semana, 9-16 de febrero, 2004: 27).

Para los promotores del proyecto, se necesitan más de cuatro años para que el presidente Uribe pueda sacar adelante su política de Seguridad Democrática (Semana, 15-22 de marzo, 2004: 46).

Su simpatía por la idea se evidenció en Estados Unidos cuando admitió que cuatro años no son suficientes para sacar adelante su plan de gobierno (Semana, 5-12 de abril, 2004: 35).

Debido a los resultados en seguridad, el empresariado considera que es crucial garantizar la continuidad de la política actual (Semana, 1926 de abril, 2004: 38).

La reelección es necesaria porque sólo con continuidad se dará respuestas a las amenazas "que tienen en jaque a la gobernabilidad democrática". Y ante la existencia de muy pocos líderes, lo mejor es alargar el periodo de los que existen (Semana, 19-26 de julio, 2004: 46).

Pero el argumento que más alienta la idea de que el futuro es incierto sin Uribe tiene que ver con la seguridad. Dentro de esta lógica, el éxito de la política de seguridad se ve atado a la permanencia del Presidente en el poder. Más cuando, en palabras del propio mandatario, "la culebra aún está viva" (Semana, 12-19 de septiembre, 2005: 41). 
Este paquete sugiere la posición de mayor respaldo a la reelección con la articulación de dos elementos significativos: la sugerencia de que debe darse la continuidad no solo de una política como la de Seguridad Democrática sino también de una persona con el perfil de Uribe Vélez, que ha obtenido resultados y se identifica como gestor y responsable de esta política, y el planteamiento de que el periodo de trabajo que el presidente tiene para llevar a cabo un conjunto de acciones exitosas que intentan solucionar un problema socialmente relevante -la seguridad- es muy corto. Con cuatro años no bastan, y mucho menos si el objetivo principal del gobierno Uribe y la política de Seguridad Democrática es acabar militarmente con el enemigo (la guerrilla).

Este paquete integra los atributos personales de Uribe Vélez con las características políticas y los logros obtenidos en materia de seguridad, mostrándolo como el mesías, como el salvador, en quien está puesta una confianza desmedida, hasta el punto de considerar que no hay un futuro posible sin él.

Por lo expuesto, en este paquete se puede ver que se torna vital la aprobación de la reelección presidencial inmediata para así permitir la continuidad de un político diferente a los demás, comprometido y trabajador, que a lo largo de su mandato ha conseguido importantes logros en materia de seguridad y que necesita cuatro años más para poder cumplir con la promesa hecha en 2002 de derrotar militarmente a la guerrilla y desarrollar por completo su plan de gobierno.

\section{d. La corrupción y el clientelismo son prácticas que caracterizan la forma de hacer política en el país; Uribe Vélez las está utilizando con el propósito de sacar adelante el proyecto de la reelección, a pesar de que su discurso se haya caracterizado por estar en contra de ellas}

El diálogo político con los contrincantes liberales o con los aliados
conservadores ha venido siendo reemplazado por una repartija buro-
crática (...), mientras el presidente siempre invita a los parlamenta-
rios a acompañarlo en sus propósitos de acabar con el clientelismo, y 
las primeras leyes las sacó sin repartir prebendas, desde que se puso a andar el proyecto de reelección inmediata, las cosas cambiaron (Semana, 2-9 de agosto, 2004: 34-36).

Como consecuencia de las presiones parlamentarias uno de los costos más altos que tuvo la aventura de la reelección para la política colombiana, y para el gobierno en particular, fue la ruptura evidente con los principios de la meritocracia que defendió el presidente Uribe en campaña. El país percibió que en aras de que la reelección pasara en el Congreso, el gobierno está repartiendo puestos a diestra y siniestra a los parlamentarios, (...) allí no sólo el presidente Uribe sino el país perdieron la oportunidad histórica de golpear una de las principales fuentes de corrupción (Pizano, Semana, 20-27 de diciembre, 2004: 18-21).

La costumbre de designar políticos o recomendados en la nómina del servicio exterior no es nueva. Ni siquiera es una práctica exclusiva de Colombia, y mucho menos es una innovación del gobierno de Álvaro Uribe (Semana, 14-21 de marzo, 2005: 25).

El gobierno Uribe, como el de sus antecesores, ha utilizado el servicio diplomático para afianzar sus apoyos en la política interna. Esta tradicional y arraigada práctica no ha cambiado en el gobierno que se considera a sí mismo "el de la antipolitiquería" (Semana, 14-21 de marzo, 2005: 25).

Las citas anteriores legitiman y justifican las acciones de Uribe Vélez con respecto al servicio exterior como botín clientelista, en la medida en que muestran la corrupción y la politiquería como una característica del quehacer político en el país. Si Uribe Vélez hace uso de estas formas tradicionales, pese a que durante parte de su gobierno buscó reivindicar la meritocracia, es con el noble propósito de sacar adelante el proyecto de reelección, proyecto que busca permitir la continuidad de la política de Seguridad Democrática.

El uso del servicio exterior por parte de Uribe Vélez como botín electoral y la reafirmación de las formas tradicionales de hacer política en el país le permiten al presidente gestionar la forma de mantenerse en el poder y así continuar con la política que pretende recuperar el orden y la seguridad y derrotar a la guerrilla. En conclusión, su vocación de trabajo por 
el país no tiene límite alguno, mucho menos si están en juego los intereses vitales de la nación.

Es por eso que la intención de salvaguardar los intereses de la patria por parte de Uribe a través del trabajo y el compromiso y de algunas medidas o acciones impopulares, como el uso del servicio exterior, son la muestra del esfuerzo de un presidente que necesita la comprensión y solidaridad del pueblo colombiano para poder solucionar los problemas del país, y más cuando se pretende por medio del proyecto de la reelección generar la posibilidad para trabajar cuatro años más.

\section{e. Con el fallo de la Corte se cambian las reglas de juego, ahora el presidente puede presentarse para ser reelegido}

La reelección, si no es de periodos consecutivos, produce el estancamiento, pero no las ventajas de la continuidad (Semana, 24-31 de octubre, 2005: 28).

Si la principal justificación de la reelección inmediata es que la prolongación de la política de Seguridad Democrática de Álvaro Uribe puede llevar a la recuperación de la soberanía, sobre todo el territorio nacional; todo este revolcón habrá valido la pena. Defender la institucionalidad es importante, pero más importante es que haya un territorio soberano donde puedan operar las instituciones (p. 29).

Por último, la historia ha demostrado que en términos generales cada vez que se abren las puertas para que pasen transformaciones audaces, después del impacto inicial, con frecuencia los resultados acaban siendo positivos a largo plazo (p. 29).

Este último paquete sugiere y condensa la posición que agrupa elementos desarrollados y presentados en paquetes anteriores, elementos que permiten y sugieren una posición frente a la figura de la reelección, como la continuidad y sus ventajas, la importancia de Uribe Vélez como gestor y ejecutor de la política de Seguridad Democrática y los resultados obtenidos y esperados de dicha política.

Luego de observar los diferentes paquetes como unidades aisladas, y con el propósito de aproximarse al tratamiento que la revista Semana le da 
a la reelección presidencial inmediata, y al observar el conjunto de los elementos y figuras que son parte de los paquetes que refuerzan el marco interpretativo, encontré que el eje principal e hilo conductor es el "rostro" de Uribe Vélez, ya que se hace presente durante todo el proceso, desde el primer planteamiento de la reelección hasta el fallo de la Corte Constitucional.

La figura de Uribe Vélez se va cultivando desde la época de campaña en 2002, en donde sembró la semilla del hombre trabajador y político diferente con la que lo identifica la mayoría de la gente. A lo largo del mandato 2002-2006 va madurando, se va afianzando en la naturaleza y el sistema político en la medida en que tenía como bandera la lucha contra la corrupción y la politiquería, terreno en el que no cede a intereses de la clase política tradicional. La política de Seguridad Democrática es el fruto del trabajo y compromiso constante, es un fruto que va floreciendo a través de los resultados.

El proceso de germinación de Uribe Vélez no había terminado y no siempre había tenido las condiciones necesarias u óptimas para propagarse, lo cual lo llevó en un momento dado a alimentarse de la clase política tradicional, que se caracteriza por hacer uso de prácticas clientelistas para alcanzar sus metas. Estas prácticas clientelistas fueron retomadas por Uribe para la gestación y desarrollo del proyecto de reelección presidencial con el fin de lograr las condiciones necesarias para que pudiera continuar madurando el principal fruto de su gobierno, la política de Seguridad Democrática, que requería continuidad.

La anterior caracterización no solo recapitula los paquetes de contenido sino que refuerza que son los ciudadanos quienes deben juzgar los mandatos presidenciales (el marco) y, en particular, que son los ciudadanos quienes deben juzgar los actos de este presidente excepcional.

Finalmente, es importante mencionar cómo se evidencia la personalización de la política. La revista Semana, al igual que la política nacional, centró el tratamiento de la reelección en una persona concreta, para este caso en Uribe Vélez, por ser este el presidente de la República y el protagonista del proyecto. La siguiente cita ejemplifica lo mencionado: 
Por más conveniente que pudiera ser la figura de la reelección inmediata, nunca habría podido ser aprobada en Colombia sin el prestigio personal de Álvaro Uribe. Si la reforma se hubiera presentado no para Uribe con nombre propio, sino para sus sucesores, nunca se habría obtenido el consenso político para sacarla adelante (Semana, 24-31 de octubre, 2005: 26-29).

Si se hace un análisis de los términos y las palabras usadas en los paquetes y en los artículos en general, es posible concluir que hay una serie de palabras que aparecen de manera repetitiva con la función de caracterizar la figura del presidente o su gobierno. A continuación se presenta una relación de las palabras con mayor frecuencia mencionadas:

\section{Tabla 1}

\section{Total de palabras repetidas}

\begin{tabular}{|c|c|}
\hline Palabra & Número de veces que se repite \\
\hline Seguridad & 27 \\
\hline Continuidad & 19 \\
\hline Cambio/desinstitucionalización & 12 \\
\hline Lucha, corrupción y politiquería & 10 \\
\hline Trabajo/gestión & 8 \\
\hline Liderazgo & 5 \\
\hline Sacrificio/compromiso & 4 \\
\hline Renovación & 2 \\
\hline Optimismo & 2 \\
\hline
\end{tabular}

Las palabras más repetidas a lo largo de los artículos están relacionadas directamente con la imagen de Uribe Vélez, ya sea haciendo referencia a la política de Seguridad Democrática o al sentimiento de seguridad que se ha desarrollado a lo largo del mandato 2002-2006:

Si hay una razón principal por la cual quieren los colombianos que Uribe se quede es por sus éxitos en materia de seguridad. La gente se siente más segura hoy y atribuye esa mejoría al empeño responsable, diario, del Presidente en velar porque la fuerza pública haga mejor su trabajo (Semana, 9-16 de febrero, 2004: 27).

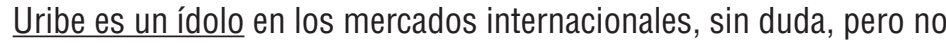
por su mentalidad, ni por la austeridad de sus políticas, sino por la 
confianza que han generado los progresos en materia de seguridad (Semana, 12-19 de septiembre, 2005: 44).

Si la principal justificación de la reelección inmediata es que la prolongación de la política de Seguridad Democrática de Álvaro Uribe puede llevar a la recuperación de la soberanía, sobre todo el territorio nacional, todo este revolcón habrá valido la pena. Defender la institucionalidad es importante, pero más importante es que haya un territorio soberano donde puedan operar las instituciones (Semana, 24-31 de octubre, 2005: 29).

Hasta ahora, la popularidad de Uribe se debe a su simpatía personal y a la percepción de que en su gobierno ha mejorado la seguridad (Semana, 24-31 de octubre, 2005: 47).

La reelección fortalece a su gran enemigo, Álvaro Uribe. Los colaboradores más cercanos del mandatario justifican su reelección como condición para culminar la tarea de derrotar a "Marulanda" y sus muchachos (Semana, 24-31 de octubre, 2005: 52).

\section{La revista Semana y la reelección presidencial inmediata en Colombia: hallazgos}

Luego de haberme aproximado al tratamiento que le dio la revista Semana al tema de la reelección presidencial inmediata, encontré una serie de elementos que permiten comprender la importancia de los medios de comunicación en la producción e intercambio de información en el sistema político, en especial en un país como Colombia, donde los medios de análisis político y opinión escasean y el interés por los asuntos políticos es cada vez menor.

La importancia de los medios de comunicación en el país radica en que por medio de ellos conocemos lo que sucede en Colombia y en el mundo, a través de ellos podemos estar al tanto de las acciones y decisiones de los gobernantes, y es a través de ellos que recibimos un alto porcentaje de los insumos que usamos para darles forma a nuestras opiniones y comportamientos políticos. Es decir, los medios no solo transmiten información, sino que influyen en la consolidación de una actitud política.

El primer hallazgo está relacionado con la posición de la revista Semana frente a la reelección presidencial. La información presentada tiende 
a apoyar la reelección, como herramienta. El marco y los paquetes de contenido están a favor de la reelección y refuerzan la figura de Uribe Vélez, dado que los artículos hacen constante referencia a términos y acciones claramente identificados con su imagen y políticas (en especial la de Seguridad Democrática, estrategia que se atribuye como el mayor logro de Uribe Vélez) y con su constante lucha contra la corrupción y la politiquería. Lo mismo ocurre con los rasgos personales que la revista identifica en la figura de Uribe Vélez, como el trabajo, el compromiso y el liderazgo. Algunos ejemplos de estos son:

La gente se siente más segura hoy y atribuye esa mejoría al empeño responsable, diario, del Presidente en velar porque la fuerza pública haga mejor su trabajo (Semana, 9-16 de febrero, 2004: 27).

Uribe es un ídolo en los mercados internacionales, sin duda, pero no por su mentalidad, ni por la austeridad de sus políticas, sino por la confianza que han generado los progresos en materia de seguridad (Semana, 12-19 de septiembre, 2005: 44).

El segundo hallazgo está directamente relacionado con el anterior, en la medida en que la revista Semana resalta la declarada lucha contra la corrupción y la politiquería por parte de Uribe Vélez, y cómo este ha podido gobernar sin ceder al juego de los políticos tradicionales, que buscan la forma de obtener beneficios personales y se caracterizan por no cumplir con las promesas al electorado. Esta presentación del presidente apoya la consolidación de la imagen de un político diferente a los demás, de un hombre de palabra que cumple lo que promete. Un ejemplo que corrobora esto es:

Gran parte de la popularidad del Presidente Uribe radica precisamente en la percepción generalizada de que este Presidente, a diferencia de sus antecesores, estaba logrando gobernar sin cederles terreno a los políticos (Semana, 9-16 de febrero, 2004: 26).

El tercer hallazgo se encuentra en la misma línea de los anteriores: la revista presenta la importancia de la política de Seguridad Democrática en el gobierno de Uribe y para el país, puesto que ha dado resultados exitosos en materia de seguridad. Semana resalta que la reelección presidencial po- 
dría beneficiar al sistema político y la cultura política del país, ya que le daría continuidad a la política de Uribe y le otorgaría cuatro años más para cumplir su promesa de derrotar a la guerrilla. Una muestra de esto es:

La reelección es necesaria porque sólo con continuidad se dará respuestas a las amenazas "que tienen en jaque a la gobernabilidad democrática". Y ante la existencia de muy pocos líderes, lo mejor es alargar el periodo de los que existen (Semana, 19-26 de julio, 2004: 46).

El cuarto hallazgo se mantiene en la línea de los anteriores en la medida en que afianza la figura de Uribe Vélez; sin embargo, esta vez la información presentada por el medio busca legitimar prácticas que caracterizan la forma de hacer política en Colombia, la corrupción y el clientelismo, y que han sido ajenas en gran parte del gobierno de Uribe, pero que están siendo empleadas para sacar adelante la reelección presidencial inmediata, justificando así una acción de gobierno.

La costumbre de designar políticos o recomendados en la nómina del servicio exterior no es nueva. Ni siquiera es una práctica exclusiva de Colombia, y mucho menos es una innovación del gobierno de Álvaro Uribe (Semana, 14-21 de marzo, 2005: 25).

El gobierno Uribe, como el de sus antecesores, ha utilizado el servicio diplomático para afianzar sus apoyos en la política interna. Esta tradicional y arraigada práctica no ha cambiado en el gobierno que se considera a sí mismo "el de la antipolitiquería" (p. 25).

El quinto hallazgo hace referencia a la posición favorable de la revista frente al fallo de la Corte con el cual se haría realidad la posibilidad de que los ciudadanos puedan juzgar a los mandatarios y que Uribe pueda ser reelegido. La posición de apoyo a la reelección ha sido defendida a lo largo del cubrimiento.

Si la principal justificación de la reelección inmediata es que la prolongación de la política de Seguridad Democrática de Álvaro Uribe puede llevar a la recuperación de la soberanía, sobre todo el territorio nacional; todo este revolcón habrá valido la pena. Defender la institucionalidad es importante, pero más importante es que haya un territorio soberano donde puedan operar las instituciones (Semana, 24-31 de octubre, 2005: 29). 
Los hallazgos mencionados previamente permiten comprender no solo el marco predominante identificado en el tratamiento que la revista Semana da a la reelección presidencial inmediata, sino también cómo un marco ayuda, persuade o influye en la forma en que las personas se acercan a un tema, lo estudian y lo recuerdan.

Un sexto hallazgo es la presentación de artículos "contextualizadores”, que se podrían llegar a ver como trabajos que buscan la objetividad que predica este medio, pero que en realidad son utilizados para no obstaculizar la posición de la revista a favor de la reelección; en otras palabras, en algunas ocasiones las revista se plantea preguntas importantes sobre posibles escenarios en Colombia, teniendo en cuenta las experiencias de otros países latinoamericanos. Por ejemplo, Semana resalta la experiencia de otros presidentes populares, que para mantenerse en el poder han permitido el crecimiento de la corrupción y han estado cerca de ir a la cárcel. Muestra de esto son:

Los ejemplos recientes del vecindario latinoamericano no son más alentadores. Carlos Menen de Argentina y Alberto Fujimori del Perú (...) El primero había hecho el milagro de devolverle la prosperidad económica al país, luego de más de una década de estancamiento. Y el segundo hizo la proeza doble, de derrotar a la guerrilla del sendero luminoso y estimular el crecimiento económico. Pero permanecer en el poder por tanto tiempo terminó en un desastre no sólo para ellos (...) sino para sus países, porque floreció la corrupción, se persiguió a los detractores y se debilitaron instituciones otrora independientes como las cortes de justicia (Semana, 9-16 de febrero, 2004: 27).

El mayor brinco a las reglas del juego que pretende dar el gobierno de Uribe es impulsar un proyecto de reelección inmediata. Esta iniciativa representa un cambio mayor de la institucionalidad colombiana. Implica no solamente que el Presidente se pueda candidatizar en 2006, sino que lo pueda hacer en condiciones de equidad con otros competidores. Para ellos se necesitaría nuevas condiciones de financiación de campañas, acceso a los medios de comunicación, permiso para que los funcionarios puedan hacer proselitismo (Semana, 2-9 de agosto, 2004: 36).

Esa prohibición tajante de negarle al pueblo la oportunidad de decidir sobre la continuidad de un gobernante tenía un sustento histórico. 
En el pasado, la experiencia negativa con los regímenes militares y gobiernos autoritarios persuadió a muchos que la mejor manera de evitar que un mandatario se perpetuara en el poder era prohibiéndolo explícitamente en la Constitución. En otras palabras, se optó por una solución legislativa a un problema político, algo habitual en la región (Semana, 24-31 de octubre, 2005: 54).

Finalmente, otro elemento que está relacionado con la contribución que hace la revista al posicionamiento de Uribe Vélez en la agenda política del país y personal de los ciudadanos, puesto que participa en su configuración como el sujeto al que hay que apostarle para el futuro, es presentarlo como la persona que modificó el panorama político del país por haber gestado el proyecto que de ahora en adelante permite a los ciudadanos juzgar los mandatos presidenciales y como la persona que ha cambiado radicalmente la seguridad y, por consiguiente, la política nacional.

\section{Referencias}

Asociación Colombiana de Investigaciones en Medios (2006). Estudio General de Medios (EGM-Colombia).

Almond, G. \& Powell, G. B. (1972). La función de la comunicación. En Política comparada: una concepción evolutiva. Buenos Aires: Paidós.

Bonilla, J. I. (2004). ¿Qué es noticia?: Agendas, periodistas y ciudadanos. Bogotá: Konrad Adenauer Stiftung.

Carragee, K. M. (2003). Evaluating polysemy: An analysis of The New York Times coverage of the end of the Cold War. Political Communication, 20, 287-308.

Cuvardic García, D. (2002). Los marcos interpretativos textuales: herramienta para el análisis del discurso periodístico. Ciencias Sociales, 2(96). Recuperado el 11 de abril de 2007, de: http: / / redalyc.uaemex.mx/redalyc/pdf/153/15309607.pdf 
Gamson, W. \& Modigliani, A. (1989). Media discourse on nuclear power: A constructionist approach. The American Journal of Sociology, 95(1), 1- 37.

Gitlin, T. (1980).Media routines and political crisis. En Gitlin, T. The whole world is watching: mass media in making and unmaking of the new left. Berkeley y Los Ángeles: University of California Press.

Gómez Giraldo, J. C. (2005). Del régimen de comunicación política en el gobierno del presidente de Colombia Álvaro Uribe Vélez: un estudio de caso (trabajo de maestría en Estudios Políticos). Pontificia Universidad Javeriana, Bogotá.

Pan, Z. \& Kosicki M., G. (1993). Framing analysis: An approach to news discourse. Political Communication, 10, 55-75.

Reese, S. (2001). Framing public life: A bridging model for media research (prólogo). En Reese. S., Gandy, Jr. O. \& Grant, A. (Eds.), Framing public life: Perspectives on media and our understanding of social world (10-11). Manhwah, NJ: Lawrence Erlbaum Associates.

Restrepo Piedrahita, C. (1988). El síndrome del presidencialismo en Colombia. Informe presentado al IV Congreso Iberoamericano de Derecho Constitucional. Madrid, 27 al 30 de Septiembre.

Santaella, M. (1990). Opinión pública e imagen política en Maquiavelo. Madrid: Alianza Editorial.

Thompson, J. (1998). Los media y la modernidad: una teoría de los medios de comunicación. Buenos Aires: Paidós.

\section{Artículos consultados de la revista Semana}

Semana (2003, 28 de julio-4 de agosto). El año que volvió la esperanza, $1108,22-28$. 
Semana (2003, 8-15 de septiembre). ¿Reelección embolatada?, 1114, 44.

Semana (2004, 2-9 de febrero). Con ganas de repetir, 1135, 40-41.

Semana (2004, 9-16 de febrero). ¿2002-2010?, 1136, 24-29.

Semana (2004, 15-22 de marzo). Agenda copada, 1141, 44-46.

Semana (2004, 5-12 de abril). La pasión de Uribe, 1144, 34-35.

Semana (2004, 19-26 de abril). 72 \% a favor, 1146, 38-43.

Semana (2004, 7-14 de junio). ¿Imparable?, 1153, 38-43.

Semana (2004, 21-28 de junio). Todos contra Uribe, 1155, 32-33.

Semana (2004, 19-26 de julio). Mano a mano, 1159, 46- 47.

Semana (2004, 2-9 de agosto). Uribe, segundo tiempo, 1161, 34-39.

Semana (2004, 1-8 de noviembre). ¿Polarizados?, 1174, 44-45.

Semana (2004, 22-29 de noviembre). La reelección no está asegurada, $1177,38-39$.

Semana (2004, 6-13 de diciembre). Cuesta arriba, 1179, 50-51.

Semana (2004, 20-27 de diciembre). La aventura de la reelección, 1181, $18-21$.

Semana (2005, 14-21 de marzo). El costo de la reelección, 1193, 24-28.

Semana (2005, 12-19 de septiembre). ¿Reelección o catástrofe?, 1219, 40-44.

Semana (2005, 24-31 de octubre). ¿Presidente o emperador?, 1225, 26-29. 
Semana (2005, 24-31 de octubre). Los secretos de la Corte", 1225, 30-35.

Semana (2005, 24-31 de octubre). El último escollo, 1225, 36-38.

Semana (2005, 24-31 de octubre). Sin sobresaltos, 1225, 40-42.

Semana (2005, 24-31 de octubre). ¿A qué juegan?, 1225, 47-48.

Semana (2005, 24-31 de octubre). Ganadores y perdedores, 1225, 52.

Semana (2005, 24-31 de octubre). El club de la reelección, 1225, 53. 